\title{
A study on the fluorescence quenching of modified $\beta$-cyclodextrin by transition metal ions in different solvents
}

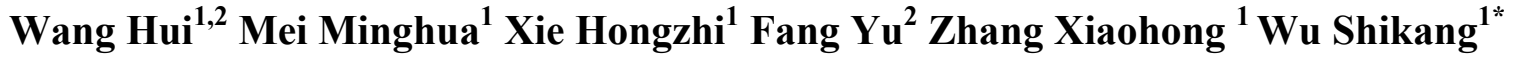 \\ ${ }^{1}$ Technical Institute of Physics and Chemistry, Chinese Academy of Sciences, \\ Beijing (100101), China. ${ }^{2}$ School of Chemistry and Material Science, Shanxi Normal University, \\ Xian (710061), China \\ E-mail: wusk@mail.ipc.ac.cn
}

\section{Dedicated to Professor Zhi-Tang Huang on his $75^{\text {th }}$ birthday}

(received 11 Feb 03; accepted 02 June 03; published on the web 06 June 03)

\begin{abstract}
The fluorescence quenching of dimethylaminochalcone modified $\beta$-cyclodextrin (DMAC-CD) by different transition metal ions in various solvents has been studied. We found that the modified $\beta$-cyclodextrin had the specific ability to recognize $\mathrm{Cu}^{2+}$ ions in the aqueous solution and the quenching constant was about one order of magnitude higher than those of other metals studied in this work. In the non-aqueous solvents, such as in DMF, the specific ability of recognizing $\mathrm{Cu}^{2+}$ ions was vanished. However, the $\mathrm{Ni}^{2+}$ ion with a planar square electronic configuration presents the highest ability for the complex formation. It could be concerned with the planar square configuration formed from the four hetero-atoms in the linking chains of modified $\beta$-cyclodextrin. In this work, we demonstrate that the spatial matching in the structure between host and guest plays a very important role in the complex formation process.
\end{abstract}

Keywords: Modified $\beta$-cyclodextrin, transition metal ion, molecular recognition, fluorescence quenching

\section{Introduction}

The investigation on the cyclodextrin (CD) derivative with fluorophore used as a sensor to detect foreign species via identifying the fluorescence intensity changes shows a profound meaning in the molecular recognition field ${ }^{1-6}$. The chemical modification through introducing the fluorophore to the $\mathrm{CD}$ not only can alter its behavior from photochemical inert to active status but also can dramatically improve its detective sensitivity. Certainly, its related applications will show a very promising future. Therefore, research on this field has attracted increasing attention in a wide range of scientific and practical interests. In our previous works ${ }^{7-8}$, a novel 6-site modified $\beta$-CD with $\mathrm{N}, \mathrm{N}$-dimethyl amino-chalcone as the fluorophore and an azaoxo-hetero- 
undecyl chain as the linker have been synthesized (DMAC-CD). The relevant photo-physical behaviors and the recognized ability respect to some foreign species have been studied as well. The obtained results were very interesting and it was found that the chalcone group was able to get into the CD cavity spontaneously in the aqueous solution. This phenomenon may attribute to the hydrophobic nature of chalcone group and the suitable connected chain length. When the chalcone group is located in the cavity of $\mathrm{CD}$, it can emit stronger fluorescence than that in the aqueous solution owing to strong solvatochromic effect from the chalcone group. If the foreign species with a very appropriate molecular size is introduced into the cavity of CD, the chalcone group will be expelled out of the cavity into the water. This can decrease the fluorescence of chalcone group intensively. Then, the detection of introduced species by CD derivatives as a sensor can be monitored through the change of fluorescence emission properties. The azaoxohetero undecyl chain connected to the rim of $\mathrm{CD}$ can form a ring-like structure at the $\mathrm{CD}$ outlet while the chalcone group was inserted into the $\mathrm{CD}$ cavity. In this case the ring-like configuration seems to be able to form a nitrogen-containing void space and could be used for capturing the transition metal cations, which will be used to form a complex. At here, we infer that the studied derivative may also be able to use as a sensor to detect the transition metal ions. It can be considered that when the CD derivative is dissolved in the dimethylformamide (DMF), the chalcone groups can be screened outside the cavity. Late on, the chain connection is capable to form a new configuration that is very different from the one in the aqueous solution. So, this is evident that the photo-physical behaviors of studied compound and their ability of complex formation with foreign ions in different solvents will be changed. The present work is focused on the fluorescence quenching of above-mentioned compound by some transition metal ions in different solvents. Some valuable results have been obtained and the explanation of the effects from the configuration change of the ligand on the complex formation has been made.

Results and Discussion

The fluorescence spectra of DMAC-CD and N, N-dimethylamino-chalcone (DMAC) in aqueous solution are shown in Fig.1. The peak wavelength in spectrum of DMAC is located at $570-580 \mathrm{~nm}$, whereas the DMAC-CD is at $\sim 560 \mathrm{~nm}$.

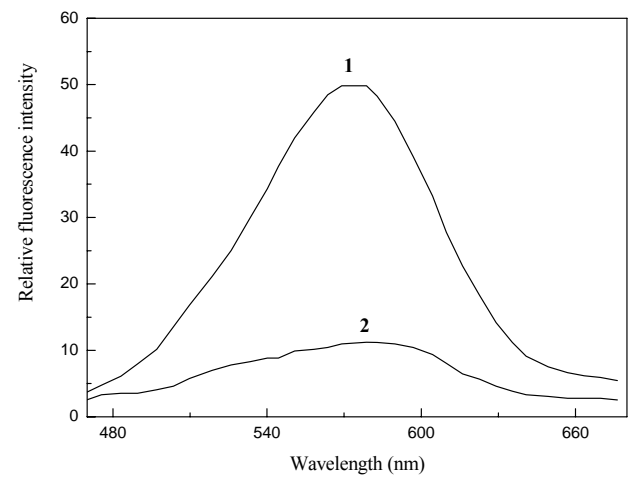

Figure 1. Fluorescence emission spectra of DMAC and DMAC-CD in aqueous solution at $\mathrm{pH}=7.0$ 1, DMAC-CD $\left(5 \times 10^{-5} \mathrm{~mol} / \mathrm{L}\right) 2$, DMAC $\left(5 \times 10^{-5} \mathrm{~mol} / \mathrm{L}\right)$. 
It can also be observed from this figure that the fluorescence intensities of both compounds are completely different, the intensity of the former peak is quite low but the later one is high. These results indicate that the chalcone part has been inserted into the cavity of CD in the aqueous solution due to the less polar environment of $\mathrm{CD}$ cavity comparing to the aqueous medium, furthermore, the solvatochromic properties ${ }^{9}$ of chalcone results in the change of the fluorescence intensity apparently. These evidences clearly demonstrate that the self-inclusion complex has been formed under this condition. In order to study the self-inclusion behaviors of compound DMAC-CD, some guest compounds are introduced as shown in Fig.2. The fluorescence intensity of DMAC-CD is dramatically decreased when these guest compounds were added in the aqueous solutions especially to the 1-adamantancarboxylic acid (1-ACA). This behavior is due to the fact that the molecular size of 1-ACA has an optimum size fitting into the cavity of the

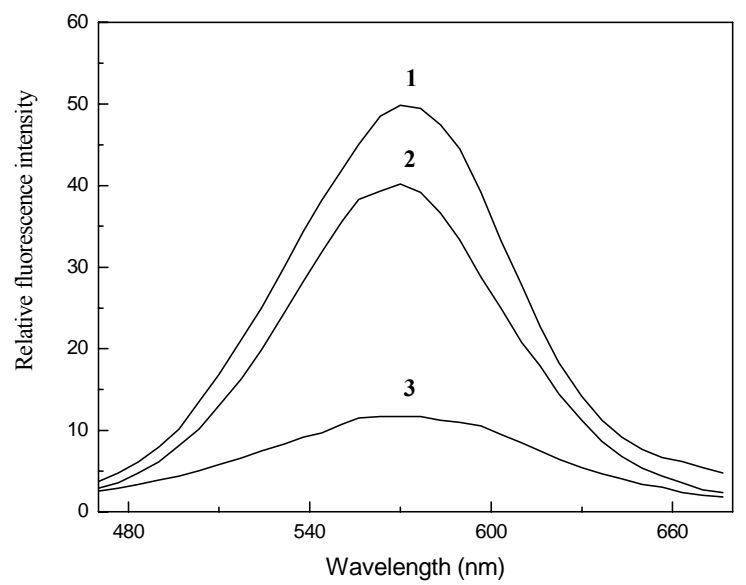

Figure 2. Fluorescence emission spectra of DMAC-CD in aqueous solution with different guest compounds 1 , in absence of guest. 2 , in presence of camphor $\left(1 \times 10^{-5} \mathrm{~mol} / \mathrm{L}\right) .3$, in presence of 1 -ACA $\left(1 \times 10^{-5} \mathrm{~mol} / \mathrm{L}\right)$.

$\beta$-cyclodextrin, moreover, the guest molecule is going to be easily driven into the CD cavity. In this case the chalcone moiety located inside of $\mathrm{CD}$ will be escaped from the cavity to the aqueous medium where the fluorescence intensity of chalcone reduced very much. These results confirm that the contained chalcone part is exactly included into the cavity of $\mathrm{CD}$ when the DMAC-CD is dissolved in the aqueous solution.

The fluorescence quenching spectra of DMAC-CD in water and DMF are presented in Figure 3 and 4, respectively. It can be found from the figures that the chalcone fluorophore has the different emission peak wavelength when the DMAC-CD is dissolved into the different solvents. In the aqueous solution, the emission peak wavelength is at the position of $570 \mathrm{~nm}$ (the chalcone group should be located in the cavity of $\mathrm{CD}$ in the aqueous solution). There is about $30 \mathrm{~nm}$ blue shift occurred when the compound is dissolved in DMF. It demonstrated that the polarity of DMF is smaller than that of CD cavity. 


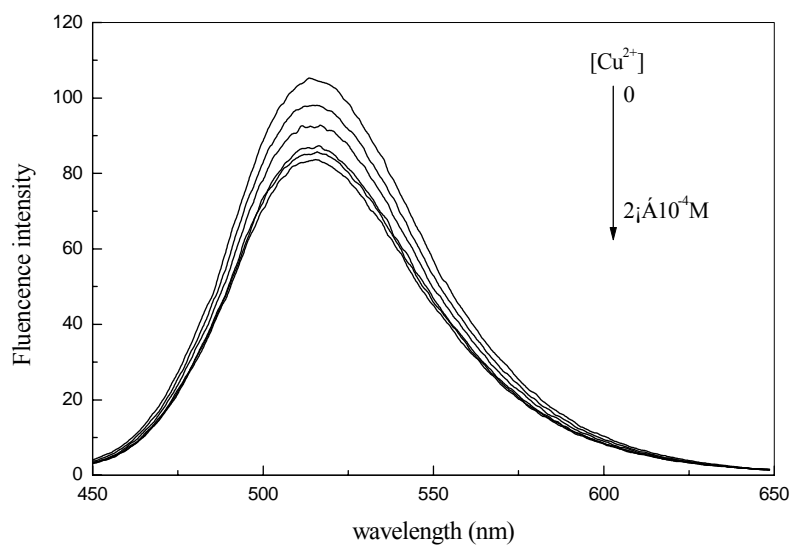

Figure 3. The emission spectra of DMAC-CD in DMF $\left(5 \times 10^{-5} \mathrm{~mol} / \mathrm{L}\right)$ in presence of $\mathrm{Cu}^{2+}$ from 0 to $2 \times 10^{-4} \mathrm{~mol} / \mathrm{L}$.

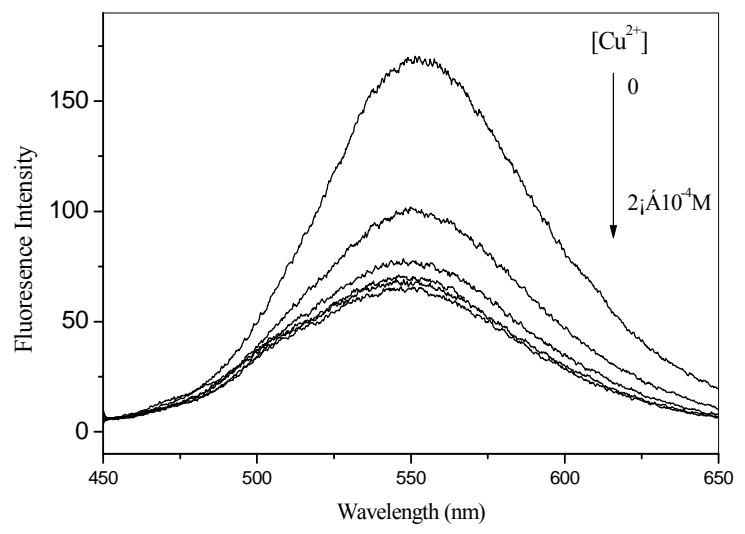

Figure 4. The emission spectra of DMAC-CD in the aqueous solution $\left(5 \times 10^{-5} \mathrm{~mol} / \mathrm{L}\right)$ in the presence of $\mathrm{Cu}^{2+}$ from 0 to $2 \times 10^{--4} \mathrm{~mol} / \mathrm{L}$.

It is interesting to find that the emission of chalcone part presents a larger quenching effect in the aqueous solution (or CD) than that in DMF when different metal ions are added. These results prove that the molecular configuration of DMAC-CD in different solvents functions as an important role for the fluorescence quenching of DMAC-CD. And, the interaction between DMAC-CD and metal ions resulted from the complex formation is assumed to strongly depend on the ligand molecular configuration very much. The fluorescence spectra of small molecule DMAC have also been recorded in the presence of transition metals. The obtained data of fluorescence quenching of DMAC present a very close quenching constant value to DMAC-CD in DMF. Hence, it indicates that the configuration of DMAC-CD is similar to that of small molecule DMAC in DMF. 
The Stern-Volmer plots of these quenching results are shown in Figure 5. There is a huge difference in the quenching behaviors, due to different quenching mechanisms.
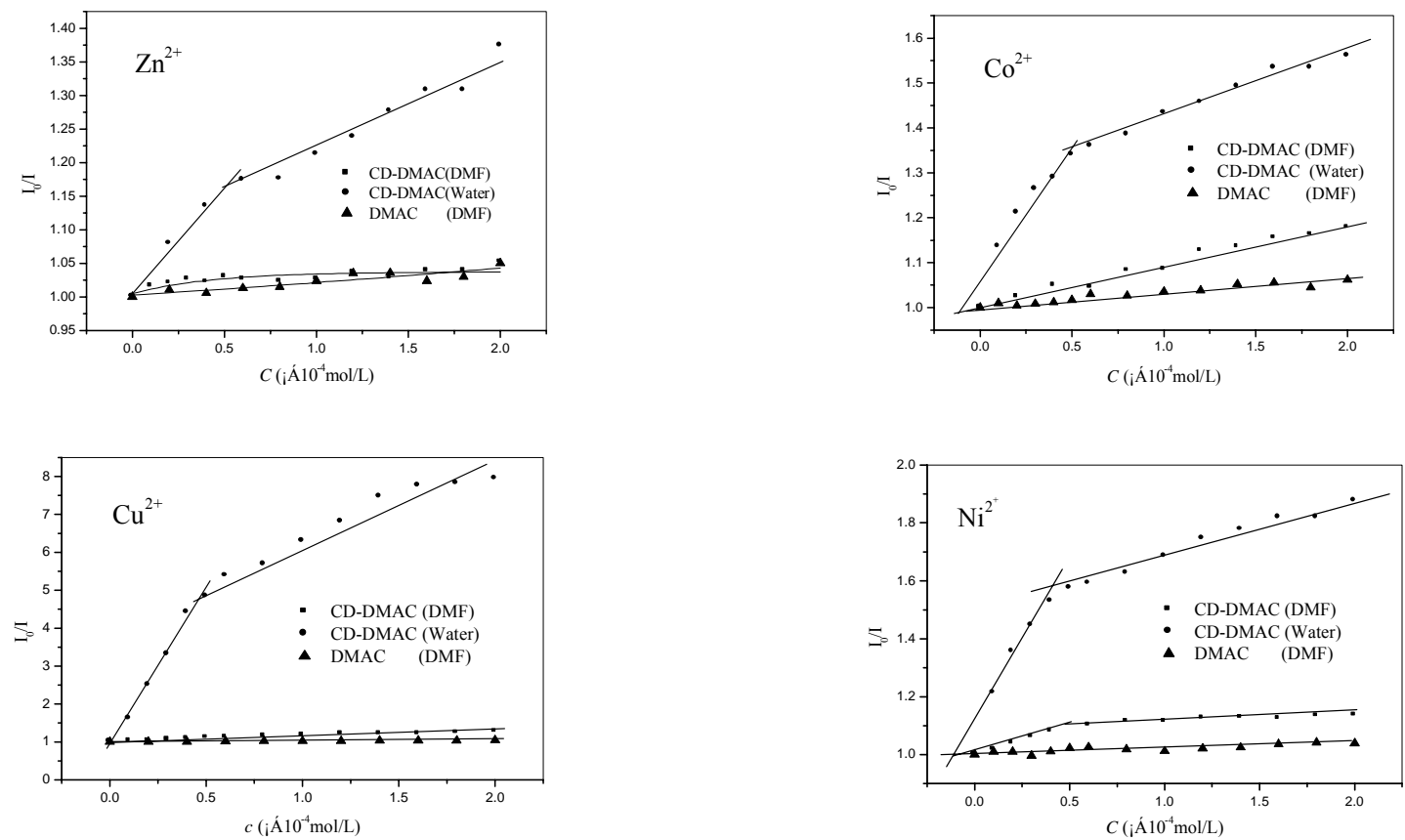

Figure 5. The Stern-Volmer plots of DMAC-CD and DMAC in different solvents, Quenchers used are $\mathrm{Zn}^{2+}, \mathrm{Co}^{2+}, \mathrm{Cu}^{2+}$ and $\mathrm{Ni}^{2+}$.

In the aqueous solution, the linked hetero-undecyl chain will be twisted to form a ring-like configuration (Scheme 1) because the chalcone group of DMAC-CD inserts into the CD cavity. In our assumption, the ring-like configuration may be favored for the complex formation with transition metal ions and resulted in a high quenching constant. On the contrary, if the chalcone group would go out from the $\mathrm{CD}$ cavity in $\mathrm{DMF}$, the ring-like structure described above is impossibly constructed and the complex formation capability would doubtlessly decreases in the meanwhile. It is clear that the configuration change of the ligand in different medium has a great impact on the complex formation capability. 


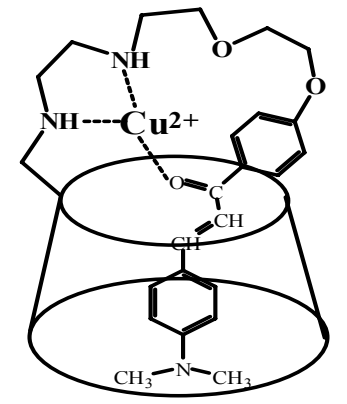

Scheme 1. The configuration of DMAC-CD when it complexes with $\mathrm{Cu}^{2+}$ in aqueous Solution.

From the Table 1, we conclude that the copper ion has the strongest quenching effect comparing to the other transitional metal ions in the aqueous solution of DMAC-CD. The quenching constant of $\mathrm{Cu}^{2+}$ ions is about one order of magnitude higher than those of the others. Obviously, the compound DMAC-CD has the highly specific recognition ability to the $\mathrm{Cu}^{2+}$ ions in the aqueous solution.

Table 1. The fluorescence lifetime and quenching constant of DMAC-CD and DMAC by different transition metal ions in different solvent

\begin{tabular}{cccccc}
\hline & $\tau(\mathrm{nm})$ & $\mathrm{Cu}^{2+}$ & $\mathrm{Co}^{2+}$ & $\mathrm{Ni}^{2+}$ & $\mathrm{Zn}^{2+}$ \\
\hline DMAC (DMF) & 1.734 & 8.97 & 18.4 & 6.07 & 0.43 \\
DMAC-CD(DMF) & 1.24 & 105.4 & 74.03 & 165.32 & 12.98 \\
DMAC-CD (Water) & 2.23 & 22500 & 5220 & 5280 & 4020 \\
DMAC-CD $\left(\mathrm{k}_{\mathrm{q}(\mathrm{H} 2 \mathrm{O})} /\right.$ & & 214 & 70 & 32 & 309 \\
$\left.\mathrm{k}_{\mathrm{q}(\mathrm{DMF})}\right)$ & & & & & \\
\hline
\end{tabular}

This phenomenon could be attributed to the good stereo-structural matching between the electronic configuration (including the suitable ionic radii) of $\mathrm{Cu}^{2+}$ ion and the active sites distribution from the formed DMAC-CD ring-like structure in the aqueous solution. For the other cations, such as $\mathrm{Ni}^{2+}, \mathrm{Co}^{2+}$ and $\mathrm{Zn}^{2+}$, the lower $\mathrm{K}_{\mathrm{q}}$ value demonstrates that there is not such good matching existed.

According to the Irving-Williams rule ${ }^{12}$, the order of stabilization constant of formed complex from different transition metal ions should be $\mathrm{Cu}^{2+}>\mathrm{Ni}^{2+}>\mathrm{Co}^{2+}$. In principle, the $\mathrm{Cu}^{2+}$ complex ought to be more stable than others. The very high stability constant of $\mathrm{Cu}^{2+}$ ion comparing to others indicates that the specific recognition of $\mathrm{Cu}^{2+}$ is truly existed for the system studied in this work. Another result observed from those data is that DMAC-CD has a higher quenching constant than that of the small molecule DMAC in DMF. The long azaoxy-heteroundecyl linking chain of DMAC-CD may be responsible for this. Although the chain of CD is able to move freely in DMF, this part can still help to bind the metal ion and draw it near to the chalcone group. Consequently, this process can greatly improve the fluorescence quenching of DMAC-CD in DMF. Meanwhile, it is also worthy to note that $\mathrm{Ni}^{2+}$ has the second strongest ability to the fluorescence quenching of DMAC-CD. This property may attribute to the planar 
square electronic configuration $\left(\mathrm{sp}^{2} \mathrm{~d}\right)$ of $\mathrm{Ni}^{2+}$ cation ${ }^{11}$. Furthermore, the $\mathrm{Ni}^{2+}$ electronic configuration can be easy to form complex with the hetero-undecyl chain part of DMAC-CD, which includes two oxygen atoms and two nitrogen atoms in DMF solution with a good static quenching effect (see Table 2).

Table 2. The lifetime of DMAC-CD in DMF with and without $\mathrm{Ni}^{2+}$

\begin{tabular}{lccc}
\hline & & $\tau(\mathrm{ns})$ & $\chi^{2}$ \\
\hline DMAC-CD & & 1.2206 & 1.135 \\
DMAC-CD: $\mathrm{Ni}^{2+}=1: 5$ & $(\mathrm{~mol} / \mathrm{mol})$ & 1.311 & 1.226 \\
DMAC-CD: $\mathrm{Ni}^{2+}=1: 0.5$ & $(\mathrm{~mol} / \mathrm{mol})$ & 1.2369 & 1.115 \\
\hline
\end{tabular}

This type of static quenching could be related to the abnormal behavior of the Stern-Volmer plot shown in this paper. When the 1:1 stoichiometric ratio is approached between the quencher and DMAC-CD, the quenching process will follow different mechanism upon to the further quencher addition to the system. As shown from the Fig. 5, there is another straight quenching line with different slope, which should be interpreted by another mechanism. The evidence for the static quenching process of DMAC-CD fluorescence by $\mathrm{Ni}^{2+}$ can be supplied from the turning point in the Stern-Volmer plot. At this point the molar concentration of DMAC-CD is just equal to the molar concentration of the added metal ions. The lifetimes of DMAC-CD in DMF with and without $\mathrm{Ni}^{2+}$ measured by single photon counting method are showed in Table 2 . These results reveal that the lifetime of DMAC-CD almost has no change either in the $\mathrm{Ni}^{2+}$ ion's presence or absence. Base on the reported results in the above, we can conclude that the quenching process is static one.

For zinc ions, in our best knowledge, it is not easy to undergo a redox reaction because of the full of electron in the $3 \mathrm{~d}$ orbital. In fact, $\mathrm{Zn}^{2+}$ ions show a very low quenching ability, which is evident from the low quenching constant of $\mathrm{Zn}^{2+}$ to the small molecule DMAC. However, the $\mathrm{Zn}^{2+}$ ions present an astonished high quenching ability comparing to the other metallic ions in the fluorescence quenching of DMAC-CD in the aqueous solution. This unique property of $\mathrm{Zn}^{2+}$ ions is contributed by its electronic tetrahedric configuration, which makes itself to be easy to insert into the ring-like structure as described previously and also close to the chalcone fluorophore. In the consequence, the quenching process became to be easily formed. So, the explanation of the low quenching constant of $\mathrm{Zn}^{2+}$ to DMAC-CD in DMF is deduced from none ring-like structure existing.

In Table 1 , it can be seen that the $\mathrm{Cu}^{2+}$ has the ratio of $\mathrm{k}_{\mathrm{q}(\text { water) }} / \mathrm{k}_{\mathrm{q}(\mathrm{DMF})}$ by 214 , but $\mathrm{Co}^{2+}, \mathrm{Ni}^{2+}$ and $\mathrm{Zn}^{2+}$ are 70, 32 and 309, respectively. This is a very interesting result. It is known that $\mathrm{Cu}^{2+}$ ion possesses the specific ability to complex with DMAC-CD. According to the quenching ratio of $\mathrm{k}_{\mathrm{q}(\text { water })} / \mathrm{k}_{\mathrm{q}(\mathrm{DMF})}$ of $\mathrm{Zn}^{2+}$ ions, $\mathrm{Zn}^{2+}$ can be also considered as a sensitive ion for the fluorescence quenching of DMAC-CD in the aqueous solution even though its absolute quenching constant is less than $\mathrm{Cu}^{2+}$. It demonstrates that $\mathrm{Zn}^{2+}$ with $\mathrm{sp}^{3}$ electronic configuration 
is suitable to insert into the ring-like structure of DMAC-CD in the aqueous solution and enforce itself to contact with the fluorophore moiety of DMAC-CD - chalcone closely. In consequence, the relative quenching ability of $\mathrm{Zn}^{2+}$ is even higher than that of $\mathrm{Cu}^{2+}$. Once again, this result indicates that the suitable configuration matching between the ligand and the metallic ion is one of the important factors in the process of complex formation.

\section{Conclusions}

The synthesized new derivative of $\mathrm{CD}$ possesses a specific ability of recognizing the $\mathrm{Cu}^{2+}$ ion in the aqueous solution. After comparing the results measured from different conditions, the configuration of this derivative in different solution is regarded as the most important factor for the transition metal cation recognition. Apparently, in the process of complex formation, the stereo-structure matching between active sites distributed by ligand and electronic configuration of center metallic ions plays a vital role in the quenching ability enhancement.

\section{Experimental Section}

The structures of studied compounds are as follows:
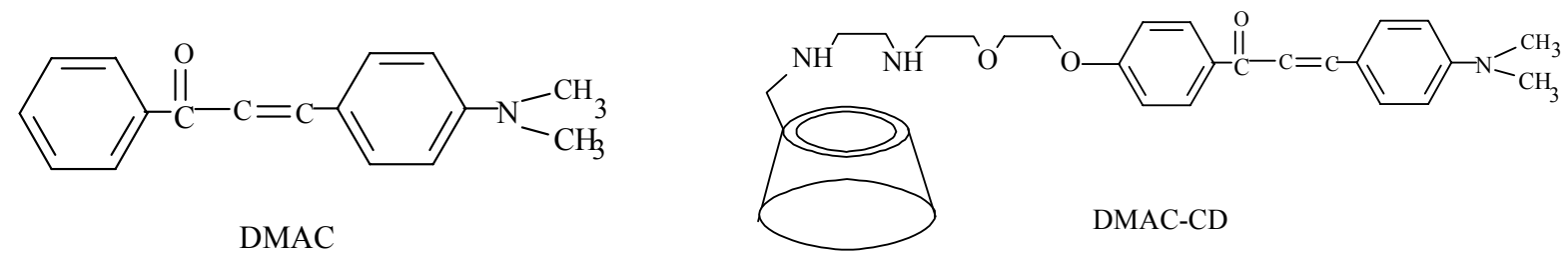

DMAC has been synthesized through the condensation of acetophenone, N, Ndimethylamino benzaldehyde. The process of synthesizing DMAC-CD is as follows: Firstly, the $\beta$-CD was reacted with p-tosyl chloride in dry pyridine. Afterward, the product - mono-6-O-6tosyl- $\beta$-cyclodextrin was purified by several times of crystallization from water, then, they react with ethylene diamine to obtain the mono-6-deoxy-(6-aminoethylamino)- $\beta$-cyclodextrin (CDen).

4-N,N-dimethylamino-4'-hydroxy-chalcone (DMACO) were obtained through the similar procedure as the DMAC synthesis. Lately, it reacted with diethylene glycol- $\alpha, \omega$-bis(p-tosyl sulfonate) to obtain the diethylene glycol- p-tosyl sulfonate chalcone and subsequently reacted with CD-en in DMF to get the raw product of DMAC-CD which is purified by column chromatography (the sephadex G-15 $(1.5 \times 40 \mathrm{~cm})$ was used as the fixing bed and water was the eluant). The detailed procedure of DMAC-CD synthesis and the characterization data can be found in reference ${ }^{7}$. Both water and DMF used as solvents in throughout experiment have been purified prior to use and were ensured that there was no any fluorescence interference existed. 
UV-Visible absorption and fluorescence spectra were recorded by Hitachi 330 spectrophotometer and Hitachi 4500 fluorescence spectrophotometer respectively. The transition metal salts used as quenchers were: zinc acetate, copper acetate, nickel chloride and cobalt acetate. These reagents were at analytical grade purchasing from Beijing Chemical Factory and they were used as received. The 1-Adamantancarboxylic acid (1-ACA) and (R)-(+) Camphor are all the products of Aldrich and used as received.

\section{Acknowledgement}

This work is supported by the "973" foundation (G2000078100). The authors would like to express thanks to Dr. G.Q. Yang from the Institute of Chemistry, the Chinese Academy of Sciences for the helping to measure the fluorescence life-time.

\section{References}

1. Breslow, R.; Overman L.E. J. Am. Chem. Soc. 1970, 92, 1075.

2. Tabushi, I. Coord. Chem. Rev. 1988, 86, 1.

3. Saenger, W. Angew. Chem. Int. Ed. Engl. 1980, 19, 344.

4. Corradini, R.; Dossena, A.; Marchelli, R.; Panagia, A.; Sartor, G.; Saviano, M.; Lombardi, A.; Pavone, V. Chem. Eur. J. 1996, 2, 373.

5. Easton, C. J.; Lincolin, S. F. Chem. Soc. Rev. 1996, 99, 163.

6. Corradini, R.; Dossena, A.; Galaverna, G.; Marchelli, R.; Panagia, A.; Sartor, G. J. Org. Chem. 1997, 62, 6283.

7. Xie Hongzhi, Wang Pengfeoi, Wu Shikang Progress in Natural Sciences (Chinese) 1999, 9, 1220.

8. Xie Hongzhi, Sun Zhaoyong, Zhang Xinkang, Wu Shikang Acta Chimica Sinica (Chinese) 2001, 59, 793.

9. Wang Pengfei, Wu Shikang J. Luminescence 1994, 62, 33.

10. Wang Pengfei, Wu Shikang Acta Chimica Sinica (Chinese) 1994, 52, 341.

11. Ci Yunxiang, Zhou Tianze The Complex in Analytical Chemistry (Chinese), Peking Univ. Press, Beijing.

12. Schwarzenbach Helv. Chim. Acta 1952, 35, 2344. 